\title{
Localization and segmentation of optic disc in retinal images using Circular Hough transform and Grow Cut algorithm
}

Muhammad Abdullah, Muhammad Moazam Fraz, Sarah A Barman

Automated retinal image analysis has been emerging as an important diagnostic tool for early detection of eye related disease such as glaucoma and diabetic retinopathy. In this paper, we have presented a robust methodology for optic disc detection and boundary segmentation, which can be seen as the preliminary step in the development of a computer-assisted diagnostic system for glaucoma in retinal images. The proposed method is based on morphological operations, Circular Hough transform and Grow Cut algorithm. The morphological operators are used to enhance the optic disc and remove the retinal vasculature and other pathologies. The optic disc center is approximated using the Circular Hough Transform, and the Grow Cut algorithm is employed to precisely segment the optic disc boundary. The method is quantitatively evaluated on five publicly available retinal image databases DRIVE, DIARETDB1, CHASE_DB1, DRIONS-DB, Messidor and one local Shifa Hospital Database. The method achieves optic disc detection success rate as $100 \%$ for these databases with the exception of $99.09 \%$ and $99.25 \%$ for the DRIONS-DB, Messidor, and ONHSD databases respectively. The optic disc boundary detection achieved an average spatial overlap of $78.6 \%, 85.12 \%, 83.23 \%, 85.1 \%, 87.93 \%, 80.1 \%$, and $86.1 \%$ respectively for these databases. This unique method has shown significant improvement over existing methods in terms of detection and boundary extraction of the optic disc. 


\section{Localization and Segmentation of Optic Disc in Retinal Images using Circular Hough Transform and Grow Cut Algorithm}

3

4 Muhammad Abdullah', Muhammad Moazam Fraz ${ }^{2}$ and Sarah A. Barman ${ }^{3}$

$5{ }^{1}$ School of Electrical Engineering and Computer Science, National University of Sciences and

6 Technology, Sector H-12, Islamabad, 44000, Pakistan.

$7 \quad{ }^{2}$ Faculty of Science Engineering and Computing, Kingston University London, United Kingdom

8

9 Corresponding Author:

10 Muhammad Moazam Fraz

11 Street Address:

12 School of Electrical Engineering and Computer Science, National University of Sciences

Email Address:

and Technology, Sector H-12, Islamabad, 44000, Pakistan.

Phone:

19

00925190852179 


\section{Abstract}

22 Automated retinal image analysis has been emerging as an important diagnostic tool for early

23 detection of eye related disease such as glaucoma and diabetic retinopathy. In this paper, we

24 have presented a robust methodology for optic disc detection and boundary segmentation, which

25 can be seen as the preliminary step in the development of a computer-assisted diagnostic system

26 for glaucoma in retinal images. The proposed method is based on morphological operations,

27 Circular Hough transform and Grow Cut algorithm. The morphological operators are used to

28 enhance the optic disc and remove the retinal vasculature and other pathologies. The optic disc

29 center is approximated using the Circular Hough Transform, and the Grow Cut algorithm is

30 employed to precisely segment the optic disc boundary. The method is quantitatively evaluated

31 on five publicly available retinal image databases DRIVE, DIARETDB1, CHASE_DB1,

32 DRIONS-DB, Messidor and one local Shifa Hospital Database. The method achieves optic disc

33 detection success rate as $100 \%$ for these databases with the exception of $99.09 \%$ and $99.25 \%$ for

34 the DRIONS-DB, Messidor, and ONHSD databases respectively. The optic disc boundary

35 detection achieved an average spatial overlap of $78.6 \%, 85.12 \%, 83.23 \%, 85.1 \%, 87.93 \%$,

$3680.1 \%$, and $86.1 \%$ respectively for these databases. This unique method has shown significant

37 improvement over existing methods in terms of detection and boundary extraction of the optic

38 disc.

39

40

41

42

43

44

45

46

47

48

49

50

51

52

53

54

55

\section{Introduction}

Digital retinal images are widely used for early detection of retinal, ophthalmic and systemic diseases because they provide a non-invasive window to the human circularity system and associated pathologies (Jack J. Kanski \& Brad Bowling 2015). Glaucoma and Diabetic Retinopathy (DR) are among major retinal disease which are the leading cause of vision loss and blindness in the working population (Federation 2013). Early detection of these disease by screening programs and subsequent treatment can prevent blindness. Computer aided diagnostic retinal image analysis is the first step in automated screening of these diseases in large population based studies (Fraz et al. 2015). The change in anatomical structures in human retina, which includes retinal vasculature, Optic Disc (OD), optic cup and retinal pathologies are the early diagnostic indicators of several diseases such as DR, Macula Edema and Glaucoma (Jack J. Kanski \& Brad Bowling 2015). Among these, the OD is the most important feature because its visual aspects are central for glaucoma detection and other lesions assessment related to DR. The important anatomical structures presented in the retinal image are shown in Figure 1. OD detection is preliminary step for glaucoma screening, which is globally the second leading cause of blindness. Moreover, it helps in the detection and localization of other retinal structures which includes the fovea, macula and estimating vascular changes (Basit \& Fraz 2015). 


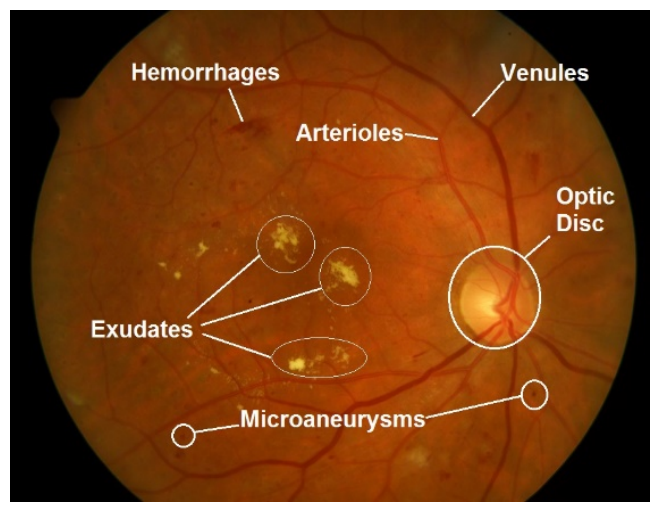

Figure 1 Important features in retinal fundus image.

Glaucoma is caused by the increase in the intraocular fluid pressure in the Optic Nerve Head $(\mathrm{ONH})$, because of either blockage or a higher production of aqueous humor of the eye (Jack J. Kanski \& Brad Bowling 2015). Glaucoma remains asymptomatic at an early stage and slowly progress with time which ultimately leads to blindness. Medical treatment is only effective at the early stages because the optic nerve, once damaged can't be cured (Weinreb et al. 2014). The early prevalence of Glaucoma can be identified by localization and segmentation of the OD and Optic Cup, followed by computing the cup-to-disc ratio. The structural changes in OD furnish critical clues pertaining to glaucoma prognosis (Abramoff et al. 2010). A Computer Assisted Diagnosis (CAD) system is necessary for large population based screening of Glaucoma. OD localization and segmentation is the first step towards the development of CAD. Knowing the significance such systems, several OD approaches have been proposed and attempted by many but it's still an active research area.

The OD appears as a variable sized, bright yellowish region, slightly oval in shape with blood vessels converging towards its center. These features are mostly used for automated OD localization. Retinal pathologies like exudates and lesions, if present, may appear like the shape of a disc, thus may cause false detection (Basit \& Fraz 2015). In CAD, accurate detection and segmentation of the OD is quite a challenging task because of various factors like boundary artifacts, missing edges and poor textural contrast. The variation in illumination conditions, luminosity and contrast during image acquisition are added challenges (Haleem et al. 2013). Moreover, the OD boundary is not constant because of the presence of incoming blood vessels which produce a fused boundary. Another distractor is papillary atrophy which, if present, appears as the bright region outside the OD and thus deforms the OD boundary (Aquino et al. 2010).

This paper presents a new approach for automatic detection and segmentation of the OD based on morphological operations, Circular Hough Transform (CHT) (Illingworth \& Kittler 1987) and Grow Cut (GC) algorithm (Vezhnevets \& Konouchine 2005). The GC algorithm has been widely used in many application areas of image segmentation, but has not been applied within the framework of retinal image analysis. To the limit of our knowledge, the GC algorithm has been utilized for the first time in localizing and segmenting the OD in retinal images. The method is evaluated on six retinal image datasets exhibiting different morphological characteristics. Experimental evaluation shows that this method is computationally fast in processing, robust to the variation in image contrast, illumination and presence of pathologies; and comparable with the state-of-the-art methodologies in terms of quantitative performance metrics.

It's worth mentioning that this work is aimed at contributing to the development of automatic systems for glaucoma detection that are currently under development. Although other published 
93

94

95

96

97

98

99

100

101

102

103

104

105

106

107

108

109

110

111

112

113

114

115

116

117

118

119

120

121

122

123

124

125

126

127

128

129

130

131

132

133

134

135

136

137

solutions can be used, this work presents higher accuracy, robustness and is tolerant to a vast variety of image characteristics, which make it suitable for integration with a glaucoma detection system.

The organization of the paper is as follows. Section 2 presents a comprehensive overview of the OD localization and segmentation methodologies available in the literature. Section 3 explains the proposed methodology in detail. The materials and the performance metrics used to evaluate the proposed methodology are illustrated in section 4 . The results and comparison with other methods are given in section 5. The paper is concluded in section 6 .

\section{Related Work}

A significant number of papers have been published to deal with the OD detection and segmentation (Haleem et al. 2013). Some papers only perform OD detection while others perform both detection and segmentation. Here we briefly discuss both of the groups.

\subsection{Methods for OD detection}

Hoover and Goldbaum (Hoover \& Goldbaum 2003) use vascular origin to detect the OD center. To detect the vascular convergence point they use a fuzzy convergence and voting type algorithm. Niemeijer et al. (Niemeijer et al. 2009) performed vascular segmentation and measure the distance at specific locations with the help of a $\mathrm{kNN}$ regressor. The point with lowest distance to the OD is selected as the OD center. Inspired by results from vascular direction methods, Youssif et al. (Youssif et al. 2008) used a matched filter to match the direction of blood vessels around the OD area and a vessel direction map is obtained by segmenting vessels. Mendonca et al.(Mendonca et al. 2013) further improve the results by using the entropy of vascular direction to assess the convergence point of vessels. To increase robustness, they constrain the search for maximal entropy to the areas with high intensities. In (Lu 2011), a circular transformation is used to capture a circular shape OD and evaluate image variation along multiple radial lines. Pixels with maximum variations are determined, as they can be further used for OD center and boundary localization. Another methodology based on the Radon transformation of overlapping window (Pourreza-Shahri et al. 2014) has achieved 100\% success in DRIVE and $96.3 \%$ on STARE databases.

\subsection{Methods for OD detection and boundary segmentation}

In (Welfer et al. 2010), a method based on mathematical morphology is proposed to detect and segment the OD in images from DRIVE and DIARETDB1. This work is extended in (Welfer et al. 2013) by incorporating a multiscale morphologic approach. Marin et al. (Marin et al. 2015) proposed a two step automatic thresholding on a morphologically processed bright enhanced region to get a reduced region of interest, followed by the application of Circular Hough Transformation (CHT) to get the OD center and OD region. Seo et al. (Seo et al. 2004) also use morphological and Canny edge detection filters to segment and detect the OD rim.

Kande et al. (Kande et al. 2008) detected the OD by using maximum local variance with 92.53\% success rate and geometric Active Contour Model (ACM) for OD segmentation. In (Aquino et al. 2010), a template based approach is used for OD segmentation. They applied morphological and edge detection techniques followed by CHT to approximate circular objects. Lupascu et al. (Lupascu et al. 2008) used a regression method and texture descriptors for circular OD fitting. An approach based on Principal Component Analysis and mathematical modelling is presented in (Morales et al. 2013), which utilizes a generalized distance function, stochastic watershed and geodesic transformations. The result is finally approximated by a circular approximation. Walter et al. (Walter et al. 2002) presents a methodology based on Watershed transformation and 
138 morphological processing. In (Hsiao et al. 2012), illumination correction technique was used to

139 detect optic disc. They select high intensity pixels as candidates for OD and among those

140 candidate pixels they select OD pixel as one with the highest variance. For segmentation, the

141 Supervised Gradient Vector Flow (SGVF) snake model is used. By extending the SGVF snake in

142 each iteration, contour points get updated and classified based on features. Statistical information

143 and features extracted from trained images were then used for classification. In (Joshi et al.

144 2011), the Chan-Vese model has been extended by introducing image information around a

145 contour point. Inspired by the work proposed in (Joshi et al. 2011), the local binary fitting

146 energy ACM (Mittapalli \& Kande 2016) is proposed to integrate the local image information

147 which includes texture color and intensity for each point of interest. A multi-resolution Sliding

148 Band Filter (SBF) was used in (Dashtbozorg et al. 2015) for OD segmentation. Super-pixels are

149 employed in (Cheng et al. 2013) such that each super-pixel is classified as OD or non-OD. It has

150

151

152

153

154 been observed that the confluence of vessels in the OD region affects the precision of OD segmentation methods. However, to overcome the influence of the presence of vessels some methods try to eliminate them from image. In this paper, we propose a new approach for automatic OD detection and segmentation which is not influenced by the confluence of vessels in $\mathrm{OD}$ area, therefore, no template or vessel map is required in advance.

\section{The Methodology}

This work presents an OD detection and segmentation methodology which is able to detect the OD center without using any template or prior vascular information, an extension to our earlier work (Abdullah \& Fraz 2015). The OD appears as a yellowish structure in retinal fundus images with shape varying from circular to slightly elliptical and has the highest intensity value pixels. However, the presence of brightness artifacts can make the OD merge into the background and lose its brightness. Furthermore, the presence of several pathological structures such as exudates may take the shape of the OD and may have the highest intensity value. The proposed algorithm is based on morphological operations, Circular Hough transform and Grow Cut algorithm. The morphological operators are used to enhance the optic disc and remove the retinal vasculature and other pathologies. The optic disc center is approximated using the Circular Hough Transform, and the Grow Cut algorithm is employed to precisely segment the optic disc boundary.

\subsection{Preprocessing}

The variation in image contrast, background illumination and pigmentation is normalized by applying pre-processing operations to the retinal images.

The green channel of an RGB image gives maximum contrast between exudates and the neighboring regions (Fraz et al. 2012b). Therefore, the green channel of RGB images is processed for normalization of contrast and luminosity. A variety of algorithms for contrast and luminosity normalization in retinal images are available in the literature, and these methodologies are either based on subtracting the estimated background from the original image (Fraz et al. 2014) or on dividing the later by the former (Foracchia et al. 2005; Vázquez et al. 2013). However, our earlier work (Fraz et al. 2014) shows that the results of both methods are similar with no appreciable advantage of one over the other. We have used the subtractive method as it has been reported in our earlier work (Fraz et al. 2014). The background pixel intensities are estimated and the difference between the estimated background and the green channel is computed to produce the normalized image. The background of the retinal image, denoted as "Ibg" is estimated by applying a filtering operation with an arithmetic mean kernel. 
183 The size of the filter kernel is not a critical parameter as long as it is large enough to ensure the 184 blurred image contains no visible structures such as optic disc, exudates or blood vessels. In this 185 work, we have used an $89 \times 89$ pixel kernel. The difference between the estimated background 186 "Ibg" and the morphologically opened image "Iopen" is calculated on pixel basis. Thus the background normalized image "Inorm" is obtained using:

$$
I_{\text {norm }}(x, y)=I_{\text {open }}(x, y)-I_{\text {bg }}(x, y)
$$

Due to different illumination conditions in the acquisition process, there may be significant intensity variations between images. These intensity variations make it difficult to use the best possible technique for all of the images, thus shade corrections were necessary and have been applied. A global linear transformation function is applied to modify the pixel intensities.

$$
I_{S C}(x, y)=\left\{\begin{array}{lr}
0 & \text { if } I_{\text {norm }}(x, y)<0 \\
1 & \text { if } I_{\text {norm }}(x, y)>1 \\
I_{\text {adjusted }}(x, y) & \text { otherwise }
\end{array}\right.
$$

$$
I_{\text {adjusted }}(x, y)=I_{\text {norm }}(x, y)-\operatorname{IntVal}_{\text {MaxPixels }}+0.5
$$

Where ${ }^{I s c}(\mathrm{x}, \mathrm{y})$ is the shade corrected image, ${ }^{I_{\text {norm }}}(\mathrm{x}, \mathrm{y})$ is the background normalized image, $\mathrm{IntVal}_{\text {MostPivels }}$ is the intensity value representing the most number of pixels in the normalized image

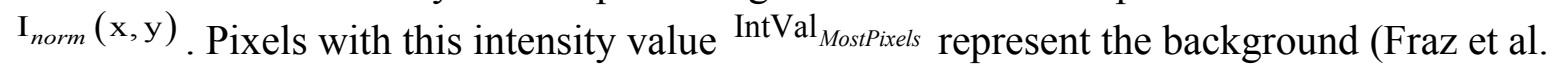
2012a). This global transformation function normalizes or shade corrects the image by setting the background pixels to 0.5, which can be observed in Figure 2(c).

\subsection{Optic Disc Detection}

After pre-processing, the OD appears as the brightest structure in the image with varying size and appearance. The retinal blood vessels originate from the OD and branch out to spread in the retinal image. A morphological closing operation with a disc shaped structuring element is applied to the pre-processed image in order to remove the vasculature from the image. The result is shown in Figure 2(d).

CHT, an extension of Hough transform (HT) (Hough 1962), is for the detection of circular objects from the image. For the detection of a circle, the HT is based on the equation of circle, defined as

$$
\left(x_{i}-a\right)^{2}+\left(y_{i}-b\right)^{2}=r^{2}
$$

Where, "( $(a, b)$ " represents the coordinates of the center of the circle and " $r$ " denotes the radius. In order to increase the performance of CHT we resize all images to a common resolution and search for the bright circles with an experimented selected radius range of 29 to 50 pixels. To avoid false detection of OD we optimized our system by applying CHT on each image at different sensitivity levels and among circular responses generated by CHT we take only strong circle. Strong circles are the ones that correspond to the OD while the rest are either exudates or misleading regions. The results of intermediary processing steps for OD localization/detection are shown in Figure 2. 


\subsection{Optic Disc Segmentation}

In the preprocessed image, the OD area is treated as foreground ( $\mathrm{fg}$ ) and the rest of the retinal image is considered as background (bg). The Grow Cut (GC) (Vezhnevets \& Konouchine 2005) algorithm separates the fg from bg using the von Neumann Neighborhood principle (Toffoli \& Margolus 1987) and seeded region growing. The detected OD center is chosen as initial seed points for fg area whereas the bg seed points are automatically chosen from rest of image. This algorithm iteratively checks each neighboring pixel and decides its region-wise membership. The GC algorithm use cellular automata for image modelling. Each image pixel "p" can be represented by a triplet (lp, $\theta p, C p)$. Where, "lp" represents the class label of the pixel "p" to which it belongs, " $\theta p$ " represents the "strength" of the pixel "p" which is a measure of the certainty of the pixel "p" that should be labelled as "lp". The label of a pixel whose "strength" = 1 cannot be changed during the algorithm progress, whereas the pixel label whose "strength" $<1$ may change during algorithm execution. "Cp" represents the pixel greyscale value.

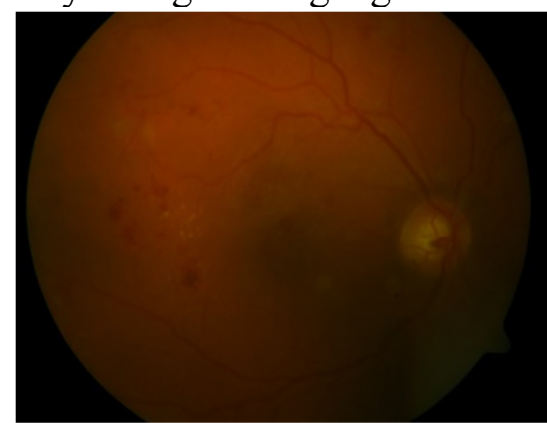

(a)

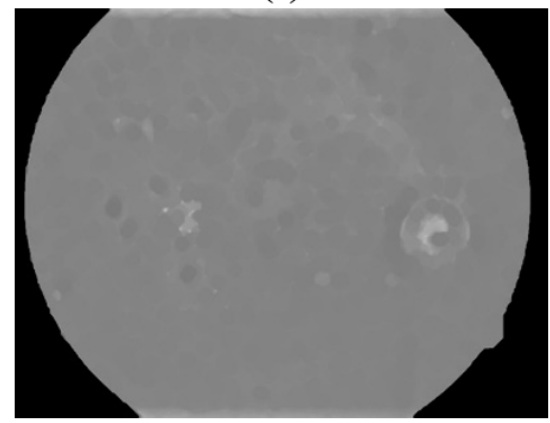

(d)

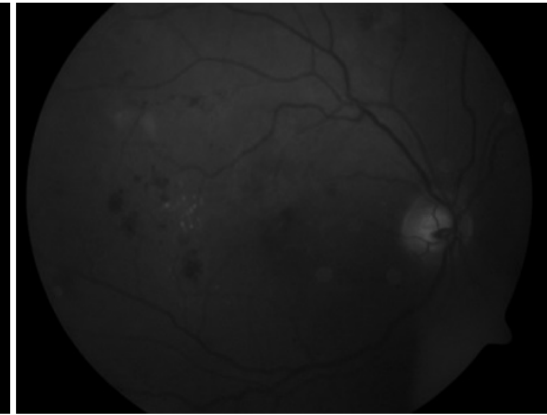

(b)

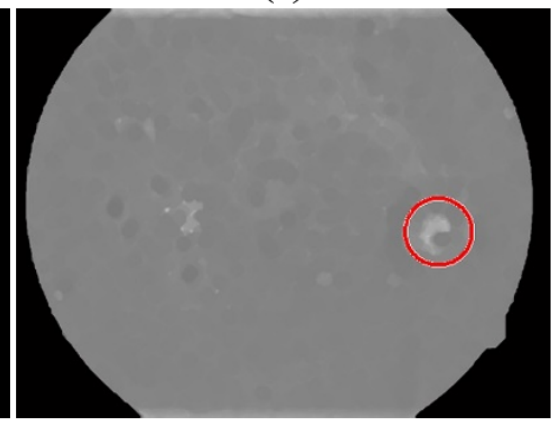

(e)

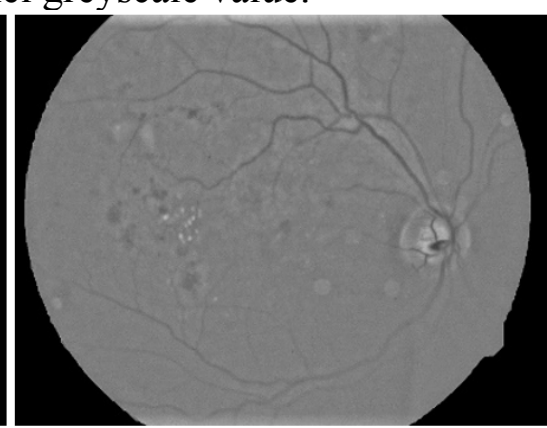

(c)

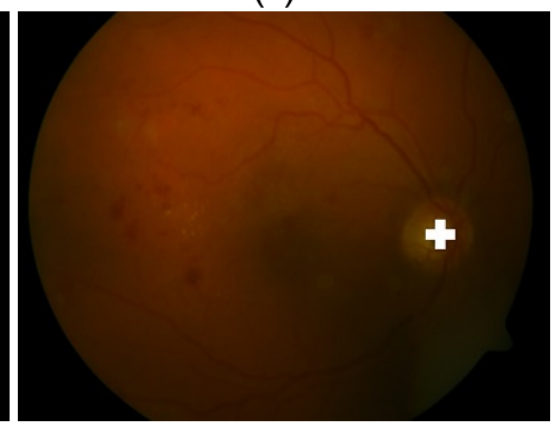

(f)

Figure 2 Processing steps for OD detection.

(a) RGB retinal image (b) Green Channel image (c) Preprocessed green channel image (d) Blood vessel removed image (e) Circular approximation of optic disc by CHT (f) Detected OD center

At the initial stage of the algorithm, the triplet for all pixels are set as,

$$
l_{p}=0, \theta_{p}=0, C_{p}=R G B_{p}
$$

Which means that initially all pixels have undefined labels and zero strength. The aim of the GC algorithm in segmenting the OD is to assign a label to each pixel in the image regarding whether it belongs to OD or to the retinal image background. To start the algorithm, we initialized seeds by setting labels for the optic disc $(+1)$ and non-OD $(-1)$. Once the seeds are initialized, the process keeps on iteratively assigning labels to each pixel in the image until all pixels are labelled. For each pixel $\mathrm{p}$ and its neighbors $\mathrm{xi}(\mathrm{i}=1$ to 8$)$, quantity "g" is computed which is monotonous decreasing function where $\mathrm{g}(\mathrm{xi}) \in[0,1]$ such that 


$$
g\left(x_{i}\right)=\frac{\left\|C_{p}-C_{x i}\right\|_{2}}{\max \|C\|_{2}}
$$

246

As we were using green channel of the image so $\left\|C_{p}-C_{x i}\right\|$ is equal to $\left\|I_{p}-I_{x_{i}}\right\|$, where ${ }^{I_{p}}$ and $I_{x_{i}}$ are the intensities of pixels $\mathrm{p}$ and xi respectively. $\max \|C\|_{2}$ is equal to $2 \mathrm{~L}-1$, where $\mathrm{L}$ is the bit depth of the image. Afterwards, the algorithm iteratively compute $\lambda$ (xi) for all pixels xi which don't have label "undefined" such that:

$$
\lambda\left(x_{i}\right)=\mathrm{g}\left(x_{i}\right) \cdot \theta\left(x_{i}\right)
$$

If $\lambda\left(x_{i}\right)>\theta_{p}$ then a pixel takes the label and strength of xi otherwise it keeps its own label and "strength". The algorithm terminates when all the pixels are labeled and the pixel label stops changing. In the end, the segmented OD boundary is approximated to an elliptical shape by using ellipse equation which involves drawing of ellipse outline over the segmented boundary of GC. The processing steps of OD boundary extraction are shown in Figure 3 . The circular approximation of the OD boundary is illustrated in Figure 4.

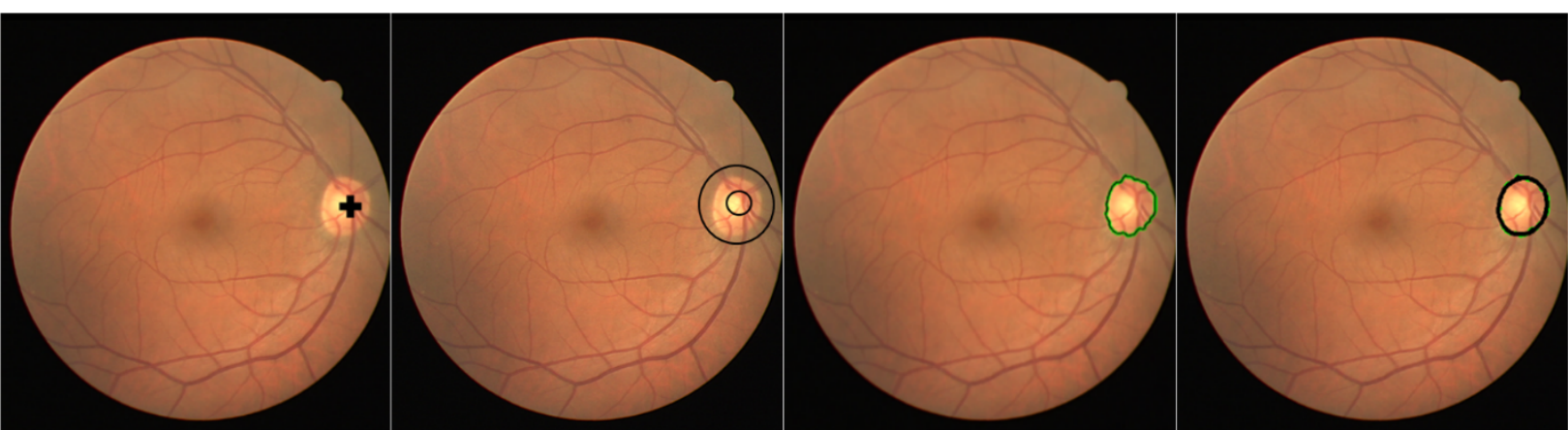

(a)

(b)

(c)

(d)

Figure 3 Steps for OD boundary extraction

(A) Original RGB image with detected central point of optic disc, (B) Foreground (optic disc area represented by small circle) selection points and background (non-optic disc region represented by large circle) selection points (C) Result of Grow Cut for boundary segmentation, (D) Boundary approximation (in black) of grow cut.

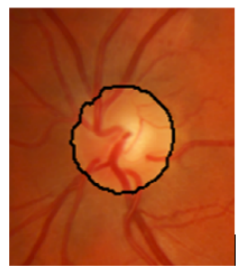

(a)

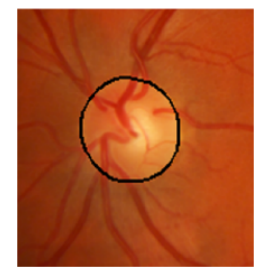

(b)

Figure 4 Close up view of (a) Grow Cut segmentation; (b) approximation of Grow Cut.

\section{Materials}

The proposed methodology is evaluated on five publicly available retinal image databases and one local database. 


\subsection{DRIVE}

269 DRIVE (2004) is a publically available database consisting of 40 images with resolution $584 \times$ 270565 pixels. Out of these 40 images, 7 are pathological, containing pigment epithelium changes, 271 exudates and hemorrhages.

$272 \quad 3.2$ DIARETDB1

273 The DIARETDB1 (Kauppi et al. 2007) database comprised 89 fundus images which are

274 obtained with a $50^{\circ}$ of FOV using a fundus camera and are in PNG format. These images are of

275 size $1500 \times 1152$ pixels, with 24 bits/pixel.

$276 \quad 3.3$ CHASEDB1

277 The CHASEDB1 (Fraz et al. 2012c) database consists of 28 images captured from a Nidek NM

$278200 \mathrm{D}$ camera at $30^{\circ} \mathrm{FOV}$. Images are of $1280 \times 960$ pixels resolution, which are affected by

279 illumination artifacts and poor contrast.

2803.4 DRIONS-DB

281 The DRIONS (Carmona et al. 2008) database consists of 110 images of $600 \times 400$ resolution, 282 with 8 bits/pixel. In these 110 images, 50 images contain some sort of defect, such as

283 illumination artifacts, rim blurredness and papillary atrophy, which may hinder the detection and 284 segmentation problem.

$285 \quad 3.5$ Messidor

286 The Messidor (Decenciere et al. 2014) database consists of 1200 retinal fundus images which 287 were captured from 3CCD color video camera on Topcon TRC NW6 non-mydriatic retinograph, 288 with $45^{\circ}$ of FOV.

2893.6 ONHSD

290 The ONHSD (Lowell et al. 2004) database consists of 99 fundus images of $640 \times 480$ resolution. 291 Images were captured from canon CR6 45MNf camera with $45^{\circ}$ of FOV. Images were acquired 292 from 50 patients, 19 out of which were diabetic.

$293 \quad 3.7$ Shifa Database

294 This database belongs to Department of Ophthalmology, Shifa International Hospital Islamabad, 295 Pakistan. 19 images are healthy while the rest of them have some sort pathological symptoms 296 and illumination artifacts. The dataset consists of 111 fundus images of $1936 \times 1296$ resolution, 297 acquired with a $45^{\circ}$ field of view.

2983.8 Ground Truths

299 The OD in all the images from the above mentioned databases is hand labelled by ophthalmic 300 experts from the Armed Forces Institute of Ophthalmology, Rawalpindi, Pakistan and used as 301 ground truths. For 1200 images in the Messidor database, we have used the ground truths 302 provided by Aquino et.al (Aquino et al. 2010). The quantitative results are based on comparison 303 of automatic segmented images with these ground truths.

304 3.9 Quantitative Performance Measures

305 The outcome of OD detection and the segmentation process results in the classification of pixels 306 belong to OD region or non-OD region. There are four possibilities for pixel classification, 307 illustrated in Table 1, True Positive (TP), True Negative (TN), False Positive (FP) and false 308 Negative (FN). The first two are the result of mutual agreement between predicted values and 309 actual values while the last two are the result of the wrong prediction. TP is the case when the 310 system predicts the pixel belongs to the OD and is actually an OD pixel in reference to the 311 ground truth image, while in the case of TN both the system and actual ground truth identify a 
312 pixel as a non-OD pixel. FP is the case where the system predicts the pixel as an OD pixel when 313 it actually belongs to non-OD region in ground truth, whereas, in the FN case the system predicts 314 a pixel as a non-OD pixel when it actually is an OD pixel.

315 The metrics used to evaluate the quantitative performance of the proposed methodology are 316 given in Table 2. We used Sensitivity (SN), Specificity (SP), Accuracy (Acc), Positive Predicted 317 Value (PPV), False Discovery Rate (FDR) and Overlap. The overlap metric is defined in (8).

$$
\text { Overlap }=\frac{\text { Area }(\text { ground truth } \cap \text { predicted })}{\text { Area }(\text { ground truth } \cup \text { predicted })}
$$

319

320

321

322

323

324

325

326

327

328

329

330

Moreover, we have used the DICE similarity index to measure the similarity between the segmented optic disc and the ground truth. The DICE index is a measurement of spatial overlap used widely for comparing segmentation results, with a value ranging from 0 to 1 . The DICE coefficient can be defined as two times the volume of the intersection between two segmentations divided by the sum of the volumes of the two segmentations, which is represented in (9).

$$
\mathrm{DICE}=\frac{2 * \text { Area }(\text { ground truth } \cap \text { predicted })}{\text { Area }(\text { ground truth })+\text { Area }(\text { predicted })}
$$

Table 1

Pixel classification

\begin{tabular}{|c|c|c|}
\hline $\begin{array}{c}\text { Real } \rightarrow \\
\text { Predicted } \downarrow\end{array}$ & $\begin{array}{c}\text { Actual pixel } \in \\
\text { OD }\end{array}$ & $\begin{array}{c}\text { Actual pixel } \notin \\
\text { OD }\end{array}$ \\
\hline System Predicted pixel $\in$ OD & TP & FP \\
\hline System Predicted pixel $\notin$ OD & FN & TN \\
\hline
\end{tabular}

Table 2

Performance metric for OD Segmentation

\begin{tabular}{|c|c|}
\hline Measure & Description \\
\hline SN & $\mathrm{TP} /(\mathrm{TP}+\mathrm{FN})$ \\
\hline $\mathrm{SP}$ & $\mathrm{TN} /(\mathrm{TN}+\mathrm{FP})$ \\
\hline Acc & $(\mathrm{TP}+\mathrm{TN}) /(\mathrm{TP}+\mathrm{FP}+\mathrm{TN}+\mathrm{FN})$ \\
\hline $\mathrm{PPV}$ & $\mathrm{TP} /(\mathrm{TP}+\mathrm{FP})$ \\
\hline FDR & $\mathrm{FP} /(\mathrm{FP}+\mathrm{TP})$ \\
\hline
\end{tabular}

\subsection{Optic Disc Detection}

The optic disc detection method achieved 100\% success rate in DRIVE, DIARETDB1, CHASE_DB1 and Shifa databases, it achieved $99.09 \%$ in DRIONS-DB and $99.25 \%$ in the Messidor database. Table 3 shows the accuracy of this method for the detection of OD. The comparison of accuracy in localizing OD have been made with other methods reported in literature in Table 4.

Table 3

Performance Measure of OD detection

\begin{tabular}{|l|l|l|l|l|}
\hline Datasets & Images & OD detected & OD missed & Accuracy \\
\hline DRIVE & 40 & 40 & 0 & $100 \%$ \\
\hline DIARETDB1 & 89 & 89 & 0 & $100 \%$ \\
\hline CHASE_DB1 & 28 & 28 & 0 & $100 \%$ \\
\hline DRIONS-DB & 110 & 109 & 1 & $99.09 \%$ \\
\hline Messidor & 1200 & 1191 & 9 & $99.25 \%$ \\
\hline Shifa & 111 & 111 & 0 & $100 \%$ \\
\hline ONHSD & 90 & 90 & 0 & $100 \%$ \\
\hline
\end{tabular}


Table 4

Comparison of OD localization with other methods

\begin{tabular}{|l|l|l|}
\hline Authors & Database & Accuracy \\
\hline (Youssif et al. 2008) & DRIVE & $98.8 \%$ \\
\hline (Niemeijer et al. 2009) & Local database & $99.4 \%$ \\
\hline (Aquino et al. 2010) & Messidor & $99 \%$ \\
\hline \multirow{2}{*}{ (Welfer et al. 2010) } & DRIVE & $100 \%$ \\
\cline { 2 - 3 } (Lu 2011) & DIARETDB1 & $97.75 \%$ \\
\hline (Zubair et al. 2013) & Messidor & $98.77 \%$ \\
\hline \multirow{2}{*}{ Mahfouz \& Fahmy 2010) } & Messidor & $98.65 \%$ \\
\hline \multirow{2}{*}{ (Yu et al. 2012) } & DRIVE & $100 \%$ \\
\hline (Saleh et al. 2014) & DIARETDB1 & $97.8 \%$ \\
\hline Proposed method & Messidor & $99 \%$ \\
\hline \multirow{5}{*}{ (2015) } & DRIVE & $100 \%$ \\
\cline { 2 - 3 } & DRIVE & $100 \%$ \\
\cline { 2 - 3 } & DIARETDB1 & $99.88 \%$ \\
\cline { 2 - 3 } & Messidor & $99.67 \%$ \\
\hline & DRIVE & $100 \%$ \\
\cline { 2 - 3 } & DIARETDB1 & $100 \%$ \\
\cline { 2 - 3 } & CHASEDB1 & $100 \%$ \\
\cline { 2 - 3 } & DRIONS-DB & $99.09 \%$ \\
\cline { 2 - 3 } & Messidor & $99.25 \%$ \\
\cline { 2 - 3 } & ONHSD & $100 \%$ \\
\hline
\end{tabular}

343

344

345

346

347

348

Figure 5 shows the results of the OD detection in the DRIVE, DIARETDB1, Shifa, CHASE_DB1 and DRIONS-DB databases. The OD detection method can correctly detect the OD center even in the presence of exudates and other pathologies. Accurate detection of the optic nerve head facilitates the segmentation algorithm to extract the boundary with high precision.

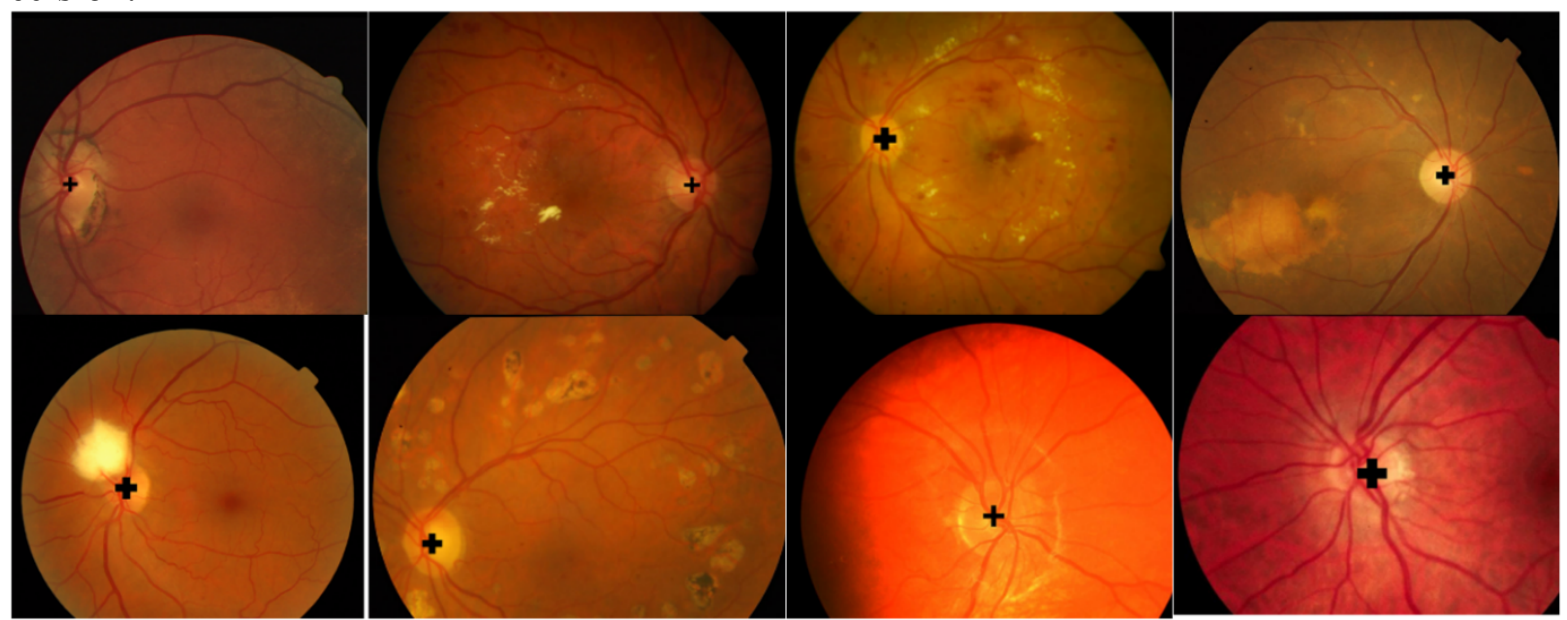

Figure 5 OD detection results. Sorting of images in rows is according to the following order DRIVE, DIARETDB1, Messidore, Shifa, CHASE_DB1 and DRIONS-DB

\subsection{Optic Disc Segmentation}

The pixel-wise quantitative performance metrics (which are defined in Table 2) are calculated for OD segmentation, based on the comparison of automatic segmented images with the ground truth reference images and are illustrated in Table 5. The methodology is quantitatively evaluated 
357 by using an array of performance metrics, which to the limit of our knowledge has not been

358

359

360

361

362

363

364

365

366

367

368

369

370

371

372

373

previously used for evaluating OD segmentation algorithms.

Table 5

Performance measures of OD segmentation

\begin{tabular}{|l|l|l|l|l|l|l|l|}
\hline $\begin{array}{l}\text { Performanc } \\
\text { e Measure }\end{array}$ & DRIVE & $\begin{array}{l}\text { DIARET } \\
\text { DB1 }\end{array}$ & $\begin{array}{l}\text { CHASE- } \\
\text { DB1 }\end{array}$ & Shifa & $\begin{array}{l}\text { DRION } \\
\text { S-DB }\end{array}$ & Messidor & ONHSD \\
\hline Acc & 0.9672 & 0.9772 & 0.9579 & 0.9793 & 0.9549 & 0.9989 & 0.9967 \\
\hline SP & 0.9966 & 0.9984 & 0.9971 & 0.9991 & 0.9966 & 0.9995 & 0.9992 \\
\hline SN & 0.8187 & 0.8510 & 0.8313 & 0.8015 & 0.8508 & 0.8954 & 0.8857 \\
\hline PPV & 0.8728 & 0.9263 & 0.9261 & 0.9493 & 0.9966 & 0.9794 & 0.9619 \\
\hline FDR & 0.1271 & 0.0737 & 0.0738 & 0.0506 & 0.0810 & 0.020 & 0.038 \\
\hline DICE & 0.8720 & 0.8910 & 0.9050 & 0.8763 & 0.9102 & 0.9339 & 0.9197 \\
\hline Overlap & $78.6 \%$ & $85.1 \%$ & $83.2 \%$ & $80.1 \%$ & $85.1 \%$ & $87.93 \%$ & $86.1 \%$ \\
\hline Hausdorff & 0.2514 & 0.1915 & 0.3174 & 0.2434 & 0.2578 & 0.1627 & 0.2245 \\
\hline
\end{tabular}

The comparison of the proposed method has been made with other available methods on the basis of average sensitivity, specificity, accuracy, DICE score, overlap, positive predictive value and the time taken to process a single image, as illustrated in

Table 6. Results shows that the proposed method provides promising results as compared to other OD segmentation techniques in the literature. The comparison with other methods is made with respect to DRIVE and DIARETDB1, DROINS-DB, Messidor and ONHSD retinal image datasets.

Table 6

Comparison of OD segmentation with other methods

\begin{tabular}{|c|c|c|c|c|c|c|c|}
\hline $\begin{array}{l}\text { Performance Measures } \rightarrow \\
\text { Methods } \downarrow\end{array}$ & Sensitivity & Specificity & Accuracy & $\begin{array}{l}\text { DICE } \\
\text { Score }\end{array}$ & $\mid \begin{array}{c}\text { Overlap } \\
\%\end{array}$ & $\begin{array}{c}\text { Predictive } \\
\text { Value }\end{array}$ & $\begin{array}{l}\text { Average } \\
\text { Time per } \\
\text { image } \\
\text { (in sec) }\end{array}$ \\
\hline \multicolumn{8}{|l|}{ DRIVE Database } \\
\hline (Sopharak et al. 2008) & 0.2104 & 0.9993 & -- & -- & 16.88 & 0.9334 & 14.92 \\
\hline (Walter et al. 2002) & 0.4988 & 0.9981 & -- & -- & 29.32 & 0.8653 & 219.6 \\
\hline (Seo et al. 2004) & 0.5029 & 0.9983 & -- & -- & 31.09 & 0.843 & 7.23 \\
\hline (Kande et al. 2008) & 0.6999 & 0.9888 & -- & -- & 29.66 & 0.5218 & 111.7 \\
\hline (Stapor et al. 2004) & 0.7368 & 0.9920 & -- & -- & 33.42 & 0.6198 & 43.00 \\
\hline (Lupascu et al. 2008) & 0.7768 & 0.9968 & -- & -- & 30.95 & 0.88 .14 & -- \\
\hline (Welfer et al. 2013) & 0.7357 & 0.9982 & -- & -- & 39.40 & 0.8876 & 53.65 \\
\hline (Basit \& Fraz 2015) & 0.8921 & 0.9921 & -- & -- & 61.88 & 0.6930 & -- \\
\hline (Morales et al. 2013) & -- & -- & 0.9903 & 0.8169 & -- & 0.8544 & -- \\
\hline (Salazar-Gonzalez et al. 2014) & 0.7512 & 0.9684 & 0.9412 & -- & -- & -- & -- \\
\hline Proposed Method & 0.8188 & 0.9966 & 0.9672 & 0.8720 & 78.6 & 0.8728 & 59.2 \\
\hline \multicolumn{8}{|l|}{ DIARETDB1 Database } \\
\hline (Sopharak et al. 2008) & 0.4603 & 0.9994 & -- & -- & 29.41 & 0.9593 & 74.55 \\
\hline (Walter et al. 2002) & 0.6569 & 0.9993 & -- & -- & 36.97 & 0.9395 & 308.5 \\
\hline (Seo et al. 2004) & 0.6103 & 0.9987 & -- & -- & 35.32 & 0.8878 & 15.63 \\
\hline (Kande et al. 2008) & 0.8808 & 0.9878 & -- & -- & 33.41 & 0.5448 & 120.5 \\
\hline
\end{tabular}




\begin{tabular}{|c|c|c|c|c|c|c|c|}
\hline (Stapor et al. 2004) & 0.8498 & 0.9964 & -- & -- & 34.08 & 0.8034 & 59.72 \\
\hline (Lupascu et al. 2008) & 0.6848 & 0.9969 & -- & -- & 30.95 & 0.8117 & -- \\
\hline (Welfer et al. 2013) & 0.6341 & 0.9981 & -- & -- & 39.15 & 0.8704 & 57.16 \\
\hline (Basit \& Fraz 2015) & 0.7347 & 0.9944 & -- & -- & 54.69 & 0.7049 & -- \\
\hline (Morales et al. 2013) & -- & -- & 0.9957 & 0.893 & -- & 0.9224 & -- \\
\hline Proposed Method & 0.851 & 0.9984 & 0.9772 & 0.891 & 85.1 & 0.9263 & 40.0 \\
\hline \multicolumn{8}{|l|}{ DRIONS-DB } \\
\hline (Walter et al. 2002) & -- & -- & -- & 0.6813 & -- & -- & -- \\
\hline (Morales et al. 2013) & -- & -- & 0.9934 & 0.9084 & -- & 0.9281 & -- \\
\hline Proposed Method & 0.8508 & 0.9966 & 0.9989 & 0.9102 & 85.1 & 0.9794 & 43.2 \\
\hline \multicolumn{8}{|l|}{ Messidor } \\
\hline (Morales et al. 2013) & -- & -- & 0.9949 & 0.8950 & -- & 0.9300 & -- \\
\hline (Kumar et al. 2015) & -- & -- & -- & 0.8456 & -- & -- & -- \\
\hline Proposed Method & 0.8954 & 0.9995 & 0.9989 & 0.9339 & 87.93 & 0.9794 & 71.3 \\
\hline \multicolumn{8}{|l|}{ ONHSD } \\
\hline (Morales et al. 2013) & -- & -- & 0.9941 & 0.8867 & -- & 0.9310 & -- \\
\hline Proposed Method & 0.8857 & 0.9992 & 0.9967 & 0.9197 & & 0.9619 & 65.3 \\
\hline
\end{tabular}

The OD segmentation results on these retinal datasets are illustrated in Figure 6 . It can be observed that the proposed methodology can successfully detect and segment the OD in the pathological images as well as in images with non-uniform illumination and uneven background pigmentation from multiple retinal image datasets.

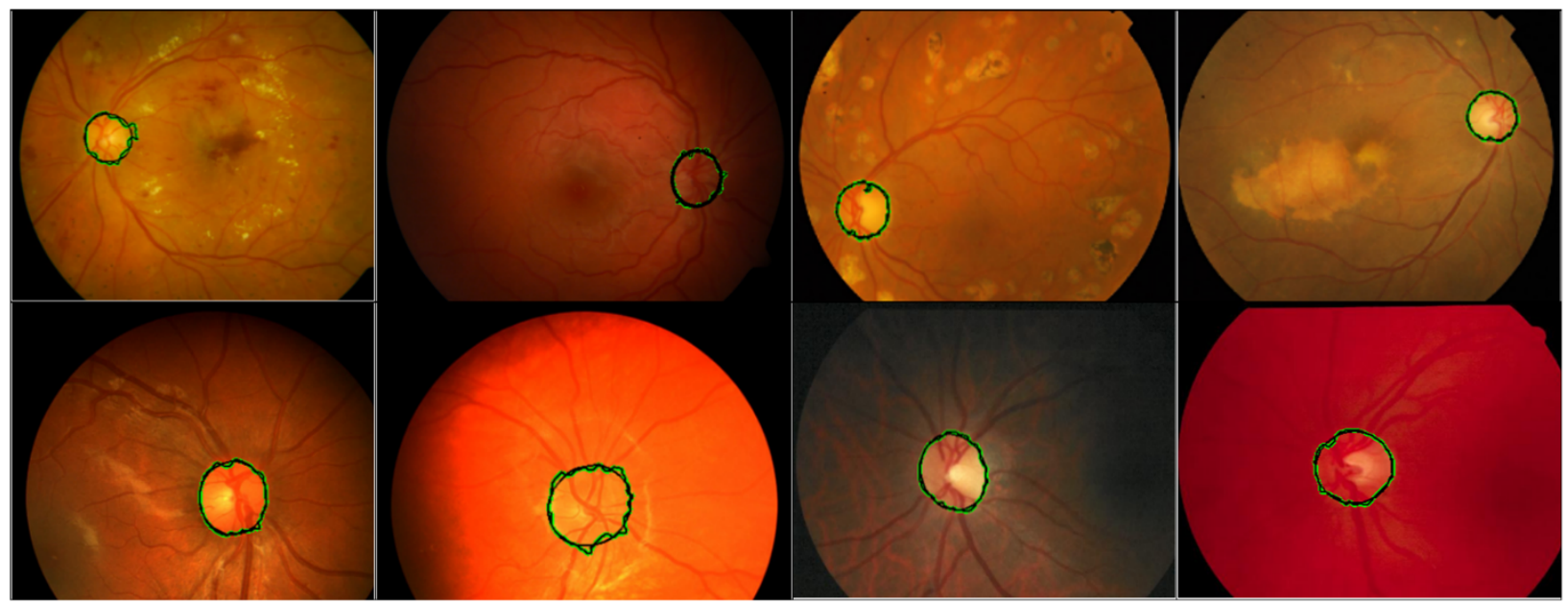

Figure 6 Examples of segmentation. Images in each row belong to separate databases as per order, DIARETDB1, Shifa, CHASE_DB1, and DRIONS-DB. Grow Cut segmentation is represented by a green boundary and its final approximation is represented by a black circle.

\subsection{Robustness of Methodology}

The robustness of proposed methodology has been evaluated on the basis of its OD localization and segmentation performance on (1) the noisy images, (2) the images with illumination artefacts, and (3) the images with pathological structures. The retinal images are corrupted with three types of noise models typically found in biomedical images (Gaussian, Salt \& Pepper, and speckle noise). It can be observed in Figure 7, that OD is successfully detected and segmented despite significant deterioration of the retinal images due to the addition of noise. 


\begin{tabular}{|c|c|c|c|}
\hline $\begin{array}{c}\text { Type of } \\
\text { noise }\end{array}$ & Original image & Noisy image & OD detection \\
\hline Gaussian & & & \\
\hline
\end{tabular}

Figure 7 Performance in the noisy images

Second criteria for measuring robustness is the evaluation of against illumination which makes OD detection harder because poor illumination hide the OD in the background, as a result of which segmentation algorithms fails to extract boundary. Figure 8 shows some extreme cases of poor illumination where proposed method successfully localize and segment the OD. 
401

402

403

404

405

406

407

408

409

410

411

412

413

414
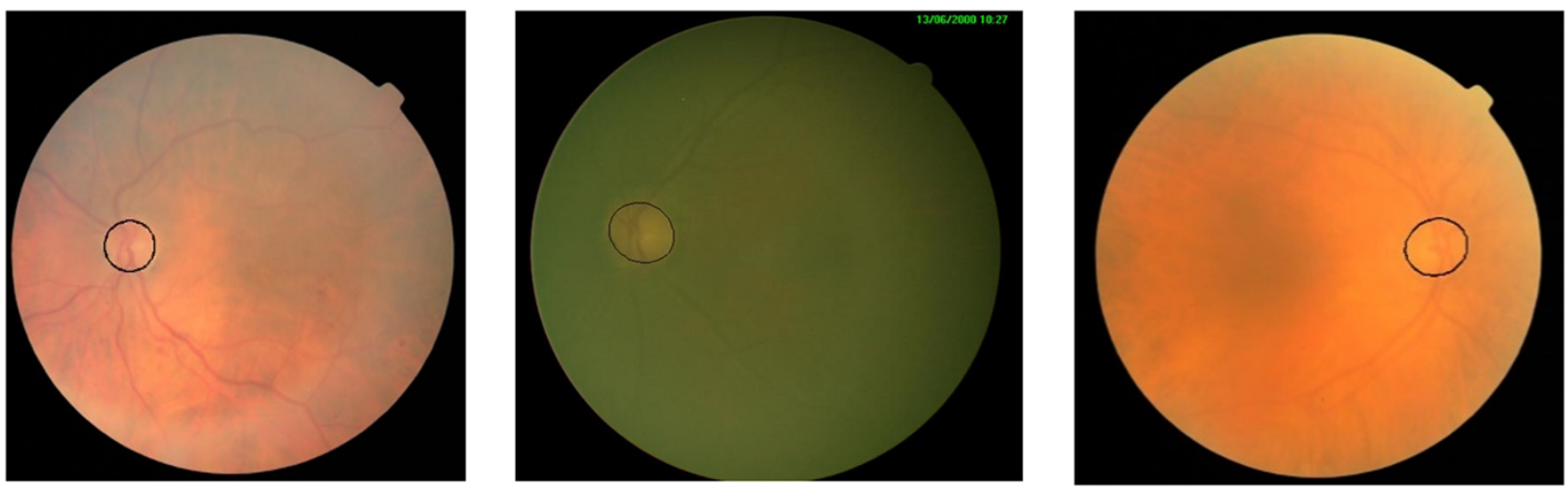

Figure 8 Performance in the poor contrast and uneven illumination.

Pathologies such as papillary atrophy, exudates, and lesions put a potent threat to accurate segmentation of optic disc because some pathologies may appear in bright or in circular shape and may result in misclassification. While others like papillary atrophy surround the OD and make it difficult to segment. Figure 9 shows the result of proposed algorithm on pathologically affected images.
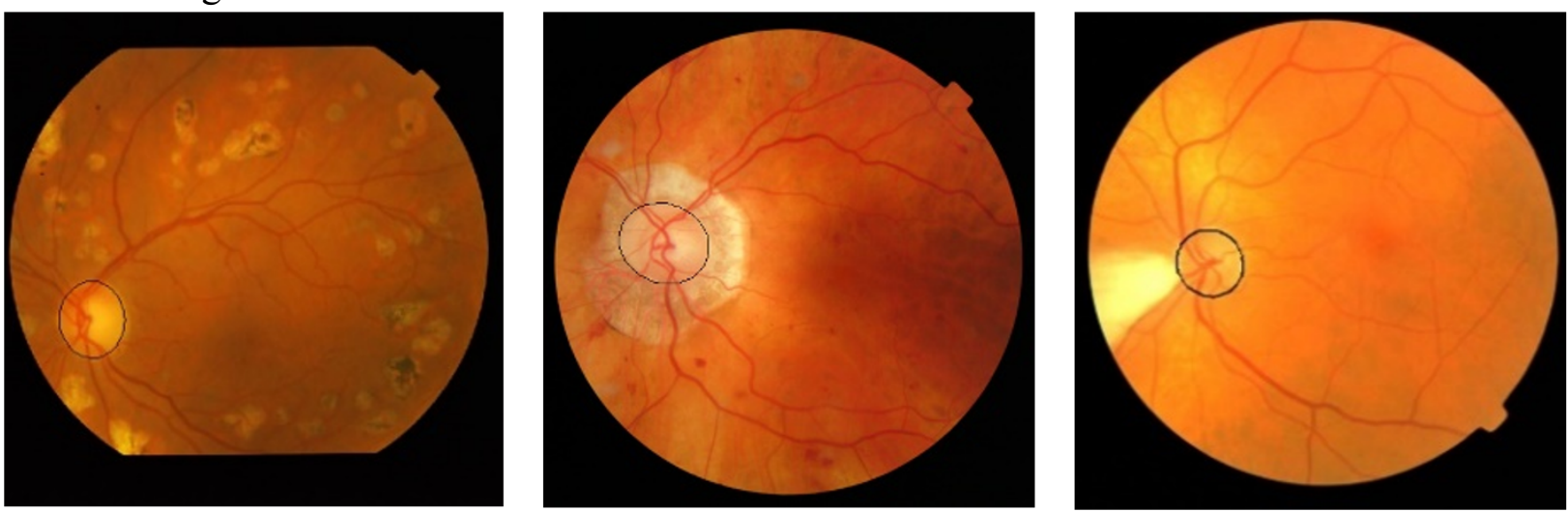

Figure 9 Performance in the presence of pathologies.

Although algorithm works fine on images where segmentation is hard but in fundus imaging, sometimes, there are images which are poorly focused or have imaging artefacts which make segmentation a difficult task.
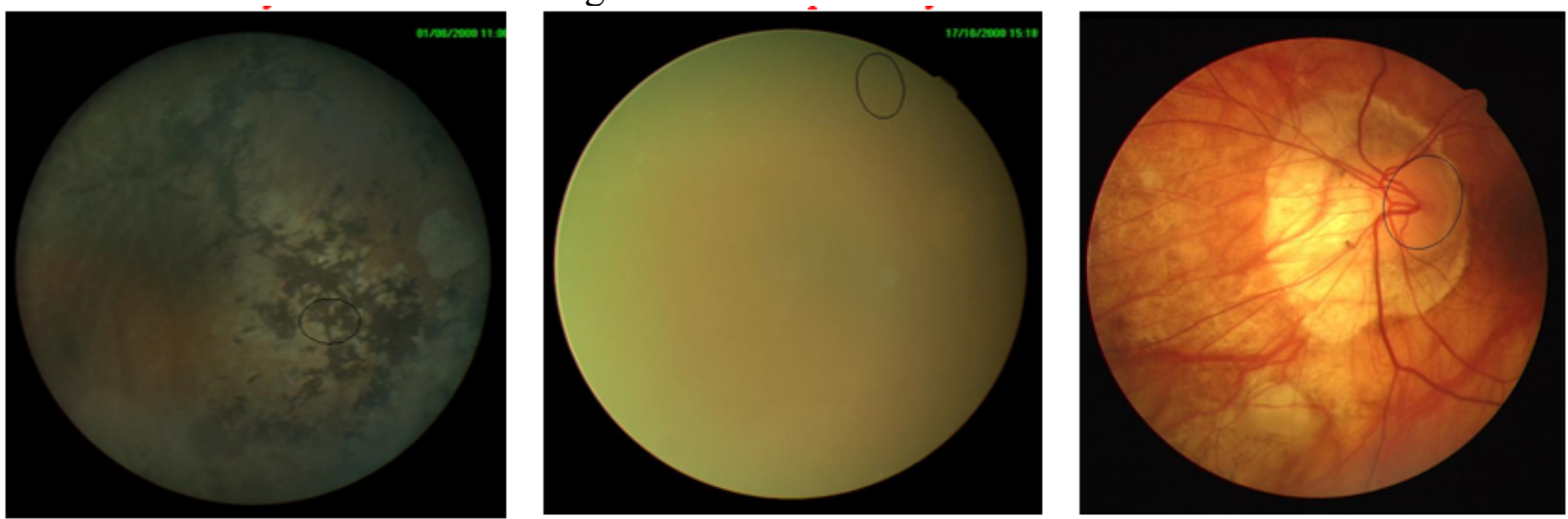

Figure 10 shows some extreme cases where method failed to extract optic disc correctly e.g. in the first two images the artifacts are so strong that it almost hide out OD and leaves false 
418 detection and segmentation while in the third image pathologies hide the boundary of OD and 419 leave the method to partially detect OD.

420

421

422

423

424

425

426

427

428

429

430

431

432

433

434

435

436

437

438

439

440

441

442

443

444

445

446

447

448

449

450

451

452
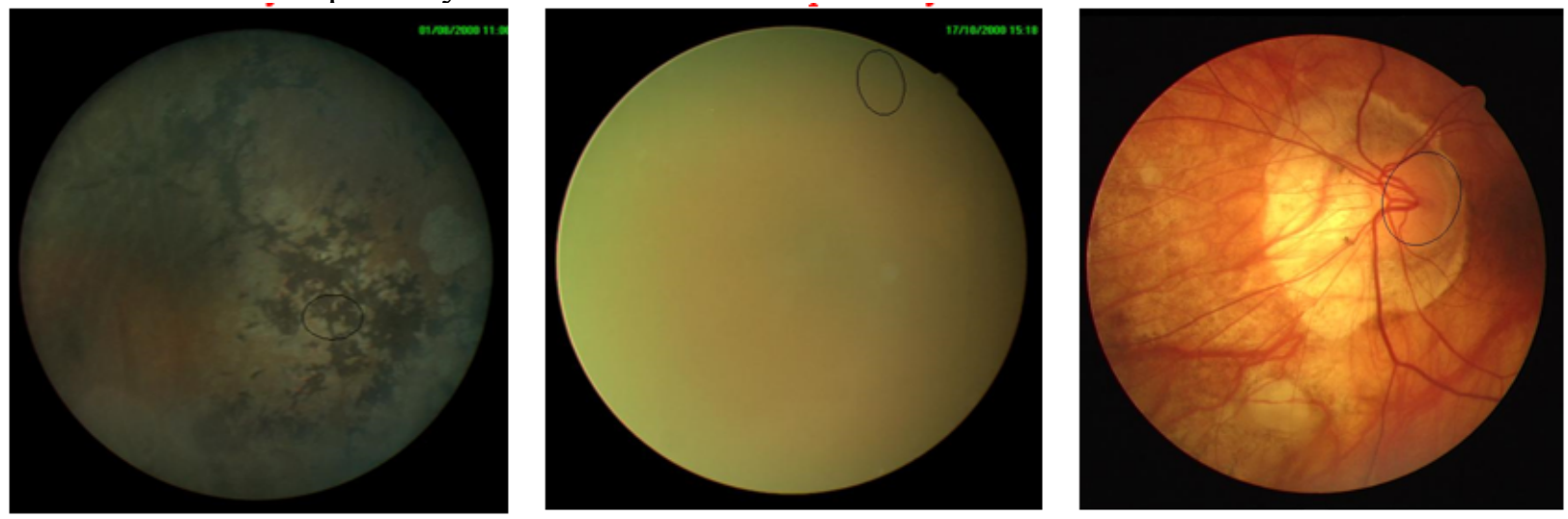

Figure 10 Incorrect OD segmentation in difficult cases of retinal images

\section{Discussion and Conclusion}

Optic disc segmentation is the primary step towards the development of automatic screening systems. The accuracy of the segmentation method improves the correct identification of pathological diseases like glaucoma. Similarly, optic disc detection is the first step towards segmentation and accurate detection would lead to promising segmentation results.

This paper presents a new method for automatic detection and segmentation of the OD in retinal images. Using morphological operations, Circular Hough Transform and Grow Cut algorithm (GC). The GC algorithm has been widely used in many application areas of image segmentation, but has not been applied within the framework of retinal image analysis. To the limit of our knowledge, the GC algorithm has been utilized for the first time in segmenting the OD in retinal images. The method is evaluated on six retinal image datasets exhibiting different morphological characteristics. Experimental evaluation shows that this method is computationally fast in processing, robust to the variation in image contrast and illumination, works well in pathological retinal images and comparable with state-of-the-art methodologies in terms of quantitative performance metrics. The methodology offers $100 \%$ OD detection rate in DRIVE, DIARETDB1, CHASE_DB1, ONHSD, and Shifa databases, and 99.09\% success rate in DRIONS-DB1. For the OD segmentation we use the detected OD center point as the seed for the Grow Cut algorithm, which then iteratively searches for neighbors of initial seeds and expands the region based on the label and strength of each pixel. The proposed method is able to segment the OD with a better overlap ratio, as compared to other methods available in the literature. We achieved $78.6 \%$, $85.1 \%, 83.2 \%, 80.1 \%, 85.1 \%, 87.93 \%$, and $86.1 \%$ in DRIVE, DIARETDB1, CHASE_DB 1 , Shifa, DRIONS-DB1, Messidor, and ONHSD databases respectively. The results of the presented algorithm can be seen online at http://vision.seecs.edu.pk/od/.

OD segmentation results clearly depict the ability of proposed method to segment, even with illumination artifacts when the OD boundary is not clear and in the presence of pathologies like papillary atrophy, which may increase the chances of false positives. The robustness of proposed methodology has been evaluated on the basis of its OD localization and segmentation performance on (1) the noisy images, (2) the images with illumination artefacts, and (3) the images with pathological structures. For evaluation purpose, the retinal images have been corrupted with three types of noises generally found in biomedical images, the Gaussian, Salt \& 
453 pepper, and Speckle noise. The methodology successfully segment the OD despite significant

454 deterioration of the retinal images. Moreover, the algorithms performs well on the images with 455 uneven illumination and the pathological structures.

456 The work is aimed at contributing to the development of an automatic system for glaucoma 457 detection that is currently under development. Although other published solutions can be used, 458 this work presents higher accuracy, robustness and is tolerant to a vast variety of images which 459 make it suitable for integration with a glaucoma detection system.

460 We have already developed a fully automated software system named QUARTZ (Fraz et al. 461 2015), which can extract a number of quantifiable measures from retinal vessel morphology. 462 These measures are analyzed/studied by epidemiologists and other medical/statistical experts in 463 464 order to evaluate the association of retinal vessel abnormalities with other systemic diseases. In future, we aim to enhance the aforementioned software system and extend its functionality by incorporating a module for early detection of glaucoma in large population based screening programs. The proposed method for reliable segmentation of OD can be seen as a first step towards the development of a glaucoma detection module.

\section{Acknowledgment}

470

471

472

473

474

475

476

477

478

479

480

481

482

483

484

485

486

487

488

489

490

491

492

493

494

495

The Authors would like to thank teams of DRIVE, DIARETDB1, CHASE_DB1, DRIONS-DB, Messidor and ONHSD for maintaining and keeping these databases active and making them easily accessible for researchers to carry out retinal image analysis research. Furthermore, we would also thank the experts from the Armed Forces Institute of Ophthalmology, Rawalpindi, Pakistan for taking the time to establish the ground truth images.

\section{References}

2004. DRIVE: Digital Retinal Images for Vessel Extraction.

Abdullah M, and Fraz MM. 2015. Application of grow cut algorithm for localization and extraction of optic disc in retinal images. 2015 12th International Conference on High-capacity Optical Networks and Enabling/Emerging Technologies (HONET): IEEE. p 1-5.

Abramoff MD, Garvin MK, and Sonka M. 2010. Retinal Imaging and Image Analysis. Biomedical Engineering, IEEE Reviews in 3:169-208. 10.1109/rbme.2010.2084567

Aquino A, Gegúndez-Arias ME, and Marín D. 2010. Detecting the optic disc boundary in digital fundus images using morphological, edge detection, and feature extraction techniques. Medical Imaging, IEEE Transactions on 29:1860-1869.

Basit A, and Fraz MM. 2015. Optic disc detection and boundary extraction in retinal images. Applied Optics 54:3440-3447. 10.1364/ao.54.003440

Carmona EJ, Rincón M, García-Feijoó J, and Martínez-de-la-Casa JM. 2008. Identification of the optic nerve head with genetic algorithms. Artificial Intelligence in Medicine 43:243-259.

Cheng J, Liu J, Xu Y, Yin F, Wong DWK, Tan N-M, Tao D, Cheng C-Y, Aung T, and Wong TY. 2013. Superpixel classification based optic disc and optic cup segmentation for glaucoma screening. Medical Imaging, IEEE Transactions on 32:1019-1032.

Dashtbozorg B, Mendonça AM, and Campilho A. 2015. Optic disc segmentation using the sliding band filter. Computers in biology and medicine 56:1-12.

Decenciere E, Zhang X, Cazuguel G, Laÿ B, Cochener B, Trone C, Gain P, Ordónez-Varela J-R, Massin P, and Erginay A. 2014. Feedback on a publicly distributed image database: the MESSIDOR database. Image Analysis and Stereology:231-234. 
496

497

498

499

500

501

502

503

504

505

506

507

508

509

510

511

512

513

514

515

516

517

518

519

520

521

522

523

524

525

526

527

528

529

530

531

532

533

534

535

536

537

538

539

540

541

Federation ID. 2013. International Diabetic Federation Atlas; Chapter 2 The Gobal Burden. Available at http://www.idf.org/sites/default/files/EN 6E Ch2 the Global Burden.pdf (accessed 3

December 2015).

Foracchia M, Grisan E, and Ruggeri A. 2005. Luminosity and contrast normalization in retinal images. Medical image analysis 9:179-190. 10.1016/j.media.2004.07.001

Fraz MM, Basit A, and Barman SA. 2012a. Application of Morphological Bit Planes in Retinal Blood Vessel Extraction. Journal of Digital Imaging:1-13. 10.1007/s10278-012-9513-3

Fraz MM, Remagnino P, Hoppe A, Uyyanonvara B, Rudnicka AR, Owen CG, and Barman SA. 2012b. Blood vessel segmentation methodologies in retinal images - A survey. Computer methods and programs in biomedicine 108:407-433.

Fraz MM, Remagnino P, Hoppe A, Uyyanonvara B, Rudnicka AR, Owen CG, and Barman SA. 2012c. An Ensemble Classification-Based Approach Applied to Retinal Blood Vessel Segmentation. Biomedical Engineering, IEEE Transactions on 59:2538-2548. 10.1109/TBME.2012.2205687

Fraz MM, Rudnicka A, Owen C, and Barman S. 2014. Delineation of blood vessels in pediatric retinal images using decision trees-based ensemble classification. International Journal of Computer Assisted Radiology and Surgery 9:795-811. 10.1007/s11548-013-0965-9

Fraz MM, Welikala RA, Rudnicka AR, Owen CG, Strachan DP, and Barman SA. 2015. QUARTZ: Quantitative Analysis of Retinal Vessel Topology and size - An automated system for quantification of retinal vessels morphology. Expert Systems with Applications 42:7221-7234. http://dx.doi.org/10.1016/j.eswa.2015.05.022

Haleem MS, Han L, van Hemert J, and Li B. 2013. Automatic extraction of retinal features from colour retinal images for glaucoma diagnosis: A review. Computerized Medical Imaging and Graphics 37:581-596.

Hoover A, and Goldbaum M. 2003. Locating the optic nerve in a retinal image using the fuzzy convergence of the blood vessels. Medical Imaging, IEEE Transactions on 22:951-958.

Hough PV. 1962. Method and means for recognizing complex patterns.

Hsiao H-K, Liu C-C, Yu C-Y, Kuo S-W, and Yu S-S. 2012. A novel optic disc detection scheme on retinal images. Expert Systems with Applications 39:10600-10606.

Illingworth J, and Kittler J. 1987. The Adaptive Hough Transform. Pattern Analysis and Machine Intelligence, IEEE Transactions on PAMI-9:690-698. 10.1109/tpami.1987.4767964

Jack J. Kanski, and Brad Bowling. 2015. Clinical Ophthalmology: A Systematic Approach. London: Elsevier Health Sciences (UK).

Joshi GD, Sivaswamy J, and Krishnadas S. 2011. Optic disk and cup segmentation from monocular color retinal images for glaucoma assessment. Medical Imaging, IEEE Transactions on 30:1192-1205.

Kande GB, Subbaiah PV, and Savithri TS. 2008. Segmentation of exudates and optic disk in retinal images. Computer Vision, Graphics \& Image Processing, 2008 ICVGIP'08 Sixth Indian Conference on: IEEE. p 535-542.

Kauppi T, Kalesnykiene V, Kamarainen J-K, Lensu L, Sorri I, Raninen A, Voutilainen R, Pietilä J, Kälviäinen $\mathrm{H}$, and Uusitalo H. 2007. DIARETDB1 diabetic retinopathy database and evaluation protocol. Medical Image Understanding and Analysis (MIUA2007). Aberystwyth, Wales, UK. p 61-65.

Kumar JH, Pediredla AK, and Seelamantula CS. 2015. Active discs for automated optic disc segmentation. 2015 IEEE Global Conference on Signal and Information Processing (GlobalSIP): IEEE. p 225-229.

Lowell J, Hunter A, Steel D, Basu A, Ryder R, Fletcher E, and Kennedy L. 2004. Optic nerve head segmentation. Medical Imaging, IEEE Transactions on 23:256-264.

Lu S. 2011. Accurate and efficient optic disc detection and segmentation by a circular transformation. Medical Imaging, IEEE Transactions on 30:2126-2133. 
542

543

544

545

546

547

548

549

550

551

552

553

554

555

556

557

558

559

560

561

562

563

564

565

566

567

568

569

570

571

572

573

574

575

576

577

578

579

580

581

582

583

584

585

586

587

Lupascu CA, Tegolo D, and Rosa LD. 2008. Automated detection of optic disc location in retinal images. Computer-Based Medical Systems, 2008 CBMS'08 21st IEEE International Symposium on: IEEE. $p$ 17-22.

Mahfouz AE, and Fahmy AS. 2010. Fast localization of the optic disc using projection of image features. Image Processing, IEEE Transactions on 19:3285-3289.

Marin D, Gegundez-Arias ME, Suero A, and Bravo JM. 2015. Obtaining optic disc center and pixel region by automatic thresholding methods on morphologically processed fundus images. Computer methods and programs in biomedicine 118:173-185.

Mendonca AM, Sousa A, Mendonca L, and Campilho A. 2013. Automatic localization of the optic disc by combining vascular and intensity information. Computerized Medical Imaging and Graphics 37:409-417.

Mittapalli PS, and Kande GB. 2016. Segmentation of optic disk and optic cup from digital fundus images for the assessment of glaucoma. Biomedical Signal Processing and Control 24:34-46.

Morales S, Naranjo V, Angulo J, and Alcañiz M. 2013. Automatic detection of optic disc based on PCA and mathematical morphology. Medical Imaging, IEEE Transactions on 32:786-796.

Niemeijer M, Abràmoff MD, and Van Ginneken B. 2009. Fast detection of the optic disc and fovea in color fundus photographs. Medical image analysis 13:859-870.

Pourreza-Shahri R, Tavakoli M, and Kehtarnavaz N. 2014. Computationally efficient optic nerve head detection in retinal fundus images. Biomedical Signal Processing and Control 11:63-73.

Salazar-Gonzalez A, Kaba D, Li Y, and Liu X. 2014. Segmentation of the Blood Vessels and Optic Disk in Retinal Images. Biomedical and Health Informatics, IEEE Journal of 18:1874-1886.

Saleh MD, Salih N, Eswaran C, and Abdullah J. 2014. Automated segmentation of optic disc in fundus images. Signal Processing \& its Applications (CSPA), 2014 IEEE 10th International Colloquium on: IEEE. p 145-150.

Seo J, Kim K, Kim J, Park K, and Chung H. 2004. Measurement of ocular torsion using digital fundus image. Engineering in Medicine and Biology Society, 2004 IEMBS'04 26th Annual International Conference of the IEEE: IEEE. $p$ 1711-1713.

Sopharak A, Uyyanonvara B, Barman S, and Williamson TH. 2008. Automatic detection of diabetic retinopathy exudates from non-dilated retinal images using mathematical morphology methods. Computerized Medical Imaging and Graphics 32:720-727. http://dx.doi.org/10.1016/i.compmedimag.2008.08.009

Stapor K, Świtonski A, Chrastek R, and Michelson G. 2004. Segmentation of fundus eye images using methods of mathematical morphology for glaucoma diagnosis. Computational Science-ICCS 2004: Springer, 41-48.

Toffoli T, and Margolus N. 1987. Cellular automata machines: a new environment for modeling: MIT Press.

Vázquez SG, Cancela B, Barreira N, Penedo MG, Rodríguez-Blanco M, Pena Seijo M, Tuero GC, Barceló MA, and Saez M. 2013. Improving retinal artery and vein classification by means of a minimal path approach. Machine Vision and Applications 24:919-930. 10.1007/s00138-012-0442-4

Vezhnevets V, and Konouchine V. 2005. GrowCut: Interactive multi-label ND image segmentation by cellular automata. proc of Graphicon: Citeseer. p 150-156.

Walter T, Klein J-C, Massin P, and Erginay A. 2002. A contribution of image processing to the diagnosis of diabetic retinopathy-detection of exudates in color fundus images of the human retina. Medical Imaging, IEEE Transactions on 21:1236-1243.

Weinreb RN, Aung T, and Medeiros FA. 2014. The pathophysiology and treatment of glaucoma: A review. JAMA 311:1901-1911. 10.1001/jama.2014.3192 
588

589

590

591

592

593

594

595

596

597

598

599

600

601

602

603

604

605

606
Welfer D, Scharcanski J, Kitamura CM, Dal Pizzol MM, Ludwig LW, and Marinho DR. 2010. Segmentation of the optic disk in color eye fundus images using an adaptive morphological approach. Computers in biology and medicine 40:124-137.

Welfer D, Scharcanski J, and Marinho DR. 2013. A morphologic two-stage approach for automated optic disk detection in color eye fundus images. Pattern Recognition Letters 34:476-485.

Youssif AA-HA-R, Ghalwash AZ, and Ghoneim AASA-R. 2008. Optic disc detection from normalized digital fundus images by means of a vessels' direction matched filter. Medical Imaging, IEEE Transactions on 27:11-18.

Yu H, Barriga ES, Agurto C, Echegaray S, Pattichis MS, Bauman W, and Soliz P. 2012. Fast localization and segmentation of optic disk in retinal images using directional matched filtering and level sets. Information Technology in Biomedicine, IEEE Transactions on 16:644-657.

$\mathrm{Yu}$ T, Ma Y, and Li W. 2015. Automatic localization and segmentation of optic disc in fundus image using morphology and level set. Medical Information and Communication Technology (ISMICT), 2015 9th International Symposium on: IEEE. p 195-199.

Zubair M, Yamin A, and Khan SA. 2013. Automated detection of Optic Disc for the analysis of retina using color fundus image. Imaging Systems and Techniques (IST), 2013 IEEE International Conference on: IEEE. p 239-242. 